\title{
The total irregularity of a graph
}

\author{
Hosam Abdo $\|^{\mathbb{*}} \quad$ Stephan Brandt $\|^{2} \quad$ Darko Dimitrov $\|^{\|}$ \\ ${ }^{1}$ Institut für Informatik, Freie Universität Berlin, Germany \\ ${ }^{2}$ Department of Mathematics and Computer Science, University of Southern Denmark, Odense, Denmark \\ received $1^{\text {st }}$ Oct. 2012, revised 10 ${ }^{\text {th }} \mathrm{Feb} .2014$, accepted $18^{\text {th }}$ Mar. 2014.
}

In this note a new measure of irregularity of a graph $G$ is introduced. It is named the total irregularity of a graph and is defined $\operatorname{as}_{\operatorname{irr}}(G)=\frac{1}{2} \sum_{u, v \in V(G)}\left|d_{G}(u)-d_{G}(v)\right|$, where $d_{G}(u)$ denotes the degree of a vertex $u \in V(G)$. All graphs with maximal total irregularity are determined. It is also shown that among all trees of the same order the star has the maximal total irregularity.

Keywords: the irregularity of a graph, the total irregularity of a graph, extremal graphs

\section{Introduction}

We consider only finite undirected graphs without loops or multiple edges. For terminology and notation not defined here we refer the reader to Bondy and Murty's textbook [6].

In this note we investigate irregularity measures of graphs. A graph is regular if all its vertices have the same degree, otherwise it is irregular. However, it is of interest to measure how irregular it is. Several approaches have been proposed that characterize how irregular a graph is.

For a graph $G$, Albertson [4] defines the imbalance of an edge $e=u v \in E(G)$ as $\left|d_{G}(u)-d_{G}(v)\right|$ and the irregularity of $G$ as

$$
\operatorname{irr}(G)=\sum_{u v \in E(G)}\left|d_{G}(u)-d_{G}(v)\right|
$$

where $d_{G}(v)$ is the degree of $v \in V(G)$. He presented upper bounds on the irregularity of graphs, bipartite graphs, and triangle-free graphs, as well as a sharp upper bound for trees. He showed that if a graph has maximal irregularity among all graphs of order $n$, then it is the join of a clique and an independent vertex set. Some claims about bipartite graphs given in [4] have been formally proved in [16]. Related to the work of Albertson is the work of Hansen and Mélot [15], who characterized the graphs with $n$ vertices

\footnotetext{
*Email: abdo@mi.fu-berlin.de

†Email: stephan.brandt@imada.sdu.dk

‡Email: darko@mi.fu-berlin.de 
and $m$ edges with maximal irregularity. For more results on imbalance, the irregularity of a graph, and other approaches, that measure how irregular a graph is, we refer the reader to [2, 3, 5, 7,-12, 17,-20].

In the sequel we introduce and consider an irregularity measure that is related to the irregularity measure (1). As well as (1), the new measure also captures the irregularity only by the difference of vertex degrees. For a graph $G$ it is defined as

$$
\operatorname{irr}_{t}(G)=\frac{1}{2} \sum_{(u, v) \in V^{2}(G)}\left|d_{G}(u)-d_{G}(v)\right| .
$$

For an ordered degree sequence of $V(G)=\left\{v_{1}, v_{2}, \ldots, v_{n}\right\}$ with $d\left(v_{1}\right) \leq d\left(v_{2}\right) \leq \ldots \leq d\left(v_{n}\right)$, (2) can be expressed in the form

$$
\operatorname{irr}_{t}(G)=\sum_{i>j}\left(d\left(v_{i}\right)-d\left(v_{j}\right)\right)
$$

Because of the obvious connection with the irregularity, we called $\operatorname{irr}_{t}(G)$ the total irregularity of a graph. Note that for every graph the total irregularity is an even number since the number of vertices of odd degree is even, and it is completely determined by its degree sequence - graphs with the same degree sequence have the same total irregularity, while this is not always true with the irregularity of a graph (see Figure 1 for such an example). Since the total irregularity of a graph depends only on its
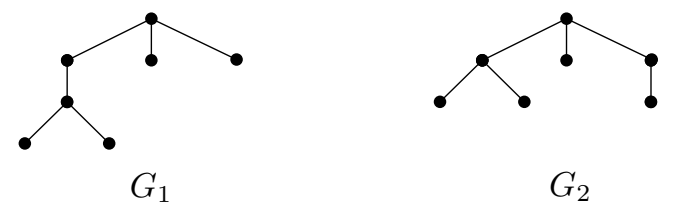

Fig. 1: Two non-isomorphic graphs $G_{1}$ and $G_{2}$ with the same degree sequence $1,1,1,1,2,3,3$. They have different irregularities $\left(\operatorname{irr}\left(G_{1}\right)=10\right.$ and $\left.\operatorname{irr}\left(G_{2}\right)=8\right)$, but the same total irregularity $\left(\operatorname{irr}_{t}\left(G_{1}\right)=\operatorname{irr}_{t}\left(G_{2}\right)=22\right)$.

degree sequence, it can be applied as irregularity measure when the adjacency information of the vertices is unknown. Another motivation to introduce $\operatorname{irr}_{t}$ as an irregularity measure is the fact that the graphs with maximal irr, among graphs of same order, are bidegreed graphs, more precisely the so-called clique-star graphs, graphs obtained by join of a clique and isolated vertices [1, 4]. Also there are a graphs with large irr that have very small degree sets, the property that one will not expect from very irregular graphs. On the contrary, as we show in this note, the graphs with maximal irr $_{t}$ have large degree sets, and some of them have even the largest possible ones.

Both measures are zero if and only if $G$ is regular, and $\operatorname{irr}_{t}(G)$ is an upper bound of $\operatorname{irr}(G)$. Very recently, these two irregularity measures were compared in [13], where it was shown that for a connected graph $G$ with $n$ vertices, $\operatorname{irr}_{t}(G) \leq n^{2} \operatorname{irr}(G) / 4$. Moreover, if $G$ is a tree, then it was shown that $\operatorname{irr}_{t}(G) \leq$ $(n-2) \operatorname{irr}(G)$.

Since the most irregular graphs with respect to irr are the join of a complete graph and an independent set, they have only two different vertex degrees. We will show below that the most irregular graphs with respect to $\operatorname{irr}_{t}$ are graphs that have many different vertex degrees, and the graphs with the maximal number of different vertex degrees belong to the graphs with maximal total irregularity. 


\section{Graphs with maximal total irregularity}

It is easy to see that there is no graph of order $n$ with $n$ different vertex degrees. Asking for graphs with $n-1$ different degrees there are two possible sets of degrees: $\{1,2, \ldots, n-1\}$ and $\{0,1, \ldots, n-2\}$ since the graph cannot contain vertices of degree 0 and $n-1$ at the same time. A simple induction proof verifies that for every $n$ there is a unique graph with the respective set of degrees up to isomorphism, see Figure 2. These graphs have been called "half-complete" in [14]. The graph with a vertex of degree $n-1$ is denoted $H_{n}$ and the graph with a vertex of degree 0 is its complement $\bar{H}_{n}$ (this can be seen by mirroring in Figure 2 one of the graphs $H_{n}, \bar{H}_{n}$ along a horizontal line). The graphs $H_{n}$ and $\bar{H}_{n}$ can be obtained by labelling the vertices $v_{1}, \ldots, v_{n}$ and adding all edges $v_{i} v_{j}$ where $i+j \geq n+1(i+j>n+1$, respectively). Note that the two vertices of $H_{n}$ with equal degrees have indices $\left\lfloor\frac{n}{2}\right\rfloor$ and $\left\lfloor\frac{n}{2}\right\rfloor+1$.
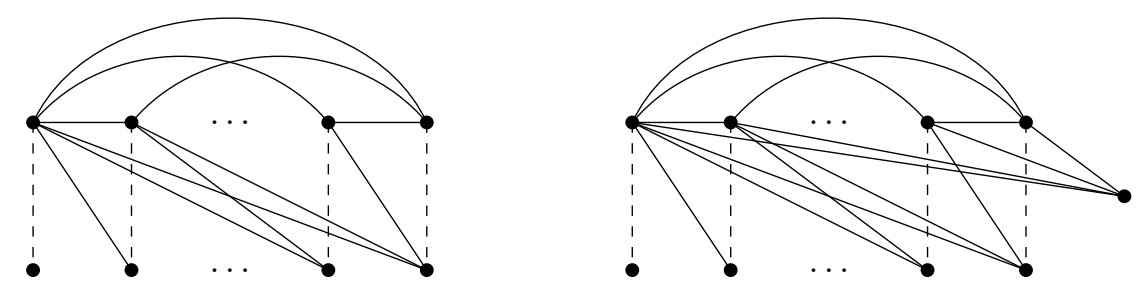

Fig. 2: The graphs $H_{n}$ (with dashed edges) and $\bar{H}_{n}$ (without dashed edges) for even and odd $n$, respectively.

Take the graph $H_{n}$ with vertices labelled as above and let $S=\left\{v_{i} v_{j} \mid i+j=n+1\right\}$, the set of dashed edges in Figure 2. Then $\bar{H}_{n}=H_{n}-S$. Now we can formulate our main result.

Theorem 2.1 A graph $G$ of order $n$ has maximal total irregularity, if and only if it is isomorphic to a graph $H_{n}-S^{\prime}$, where $\emptyset \subseteq S^{\prime} \subseteq S$.

Observe that the set $S^{\prime}$ of $G=H_{n}-S^{\prime}$ can be determined only from the degree sequence of $G$. This follows from the fact that the vertex labelling of $G$ inherited from $H_{n}$ has the property that $d_{G}\left(v_{1}\right) \leq$ $d_{G}\left(v_{2}\right) \leq \ldots \leq d_{G}\left(v_{n}\right)$. Therefore for $j<i, i+j=n+1$, we have $v_{i} v_{j} \in S^{\prime}$ if and only if $d_{G}\left(v_{j}\right)=j-1$. In particular, every two graphs $G_{1}=H_{n}-S_{1}$ and $G_{2}=H_{n}-S_{2}$ with $\emptyset \subseteq S_{1} \neq S_{2} \subseteq S$ are not isomorphic. Therefore, since $|S|=\left\lfloor\frac{n}{2}\right\rfloor$ there are exactly $2\left\lfloor\frac{n}{2}\right\rfloor$ non-isomorphic graphs of order $n$ with maximal total irregularity.

Note that all these graphs have the property that they are subgraphs of $H_{n}$ and supergraphs of $\bar{H}_{n}$, but the converse does not hold. E.g., the path on four vertices is a subgraph of $H_{4}$ and a supergraph of $\bar{H}_{4}$, but its total irregularity is 4 , while the maximal total irregularity is 6 .

Proof: For an ordered degree sequence, consider an edge $v_{i} v_{j}, i>j$. Adding $v_{i} v_{j}$ to $G$ or deleting $v_{i} v_{j}$ from $G$ only changes the summands $d(u)-d(w)$ in 3 where $\left|\{u, w\} \cap\left\{v_{i}, v_{j}\right\}\right|=1$. Adding the edge $v_{i} v_{j}$ to $G$ increases all summands $d\left(v_{i}\right)-d\left(v_{k}\right)$ with $i>k$ and $i \neq j$ by one while the summands $d\left(v_{k}\right)-d\left(v_{i}\right)$ for $k>i$ are decreased by one (or increased by one, if $d_{G}\left(v_{k}\right)=d_{G}\left(v_{i}\right)$ ). Similarly, all summands $d\left(v_{j}\right)-d\left(v_{k}\right)$ with $j>k$ are increased by one, while the summands $d\left(v_{k}\right)-d\left(v_{i}\right)$ for $k>i$ 
and $j \neq i$ are decreased by one (or increased by one, if $d_{G}\left(v_{k}\right)=d_{G}\left(v_{j}\right)$ ). Therefore,

$$
\begin{aligned}
\operatorname{irr}_{t}\left(G+v_{i} v_{j}\right) & \geq \operatorname{irr}_{t}(G)+(i-2)-(n-i)+(j-1)-(n-j-1) \\
& =\operatorname{irr}_{t}(G)+2(i+j-1)-2 n
\end{aligned}
$$

which exceeds $\operatorname{irr}_{t}(G)$ if $i+j>n+1$.

Deleting an edge $v_{i} v_{j}$ from $G$ has the effect that all summands $d\left(v_{k}\right)-d\left(v_{i}\right)$ with $k>i$ are increased by one while the summands $d\left(v_{i}\right)-d\left(v_{k}\right)$ with $k<i, k \neq j$ are decreased by one (or increased by one, if $\left.d_{G}\left(v_{k}\right)=d_{G}\left(v_{i}\right)\right)$. All summands $d\left(v_{k}\right)-d\left(v_{j}\right)$ with $k>j, k \neq i$, are increased by one, while the summands $d\left(v_{j}\right)-d\left(v_{k}\right)$ with $k<j$, are decreased by one (or increased by one, if $d_{G}\left(v_{k}\right)=d_{G}\left(v_{j}\right)$ ). Therefore,

$$
\begin{aligned}
\operatorname{irr}_{t}\left(G-v_{i} v_{j}\right) & \geq \operatorname{irr}_{t}(G)+(n-i)-(i-2)+(n-j-1)-(j-1) \\
& =\operatorname{irr}_{t}(G)+2 n-2(i+j-1)
\end{aligned}
$$

with equality if and only if $d_{G}\left(v_{j}\right)>d_{G}\left(v_{j-1}\right)$ and $d_{G}\left(v_{i}\right)>d_{G}\left(v_{i-1}\right)\left(d_{G}\left(v_{i}\right)>d_{G}\left(v_{i-2}\right)\right.$ if $\left.j=i-1\right)$. Therefore, if $G$ has maximal irregularity then $G$ has all edges $v_{i} v_{j}$ with $i+j>n+1$ and no edge with $i+j<n+1$. Any such graph can be expressed in the form $G=H_{n}-S^{\prime}$ with $\emptyset \subseteq S^{\prime} \subseteq S$. It is left to show that every such graph has the same total irregularity. Let $G=H_{n}-S^{\prime}$ where $\emptyset \subseteq S^{\prime} \subseteq S$ and let $v_{i} v_{j} \in S \backslash S^{\prime}$. Then $v_{i}$ and $v_{j}$ have both their original degree from $H_{n}$, but $d\left(v_{i-1}\right)$ and $d\left(v_{j-1}\right)$ can only have decreased. Since $d_{H_{n}}\left(v_{j}\right)>d_{H_{n}}\left(v_{j-1}\right)$ and $d_{H_{n}}\left(v_{i}\right)>d_{H_{n}}\left(v_{i-1}\right)$ if $j<i-1$ and $d_{H_{n}}\left(v_{i}\right)=$ $d_{H_{n}}\left(v_{j}\right)>d_{H_{n}}\left(v_{j-1}\right)$ if $j=i-1$, the inequality (4) holds with equality and $\operatorname{irr}_{t}\left(G-v_{i} v_{j}\right)=\operatorname{irr}_{t}(G)$.

Corollary 2.2 For any simple undirected graph $G$ with $n$ vertices it holds that

$$
\operatorname{irr}_{t}(G) \leq \begin{cases}\frac{1}{12}\left(2 n^{3}-3 n^{2}-2 n+3\right) & n \text { odd }, \\ \frac{1}{12}\left(2 n^{3}-3 n^{2}-2 n\right) & n \text { even } .\end{cases}
$$

Proof: The bounds follow with Theorem 2.1 from the total irregularity of $H_{n}$. The only two vertices of $H_{n}$ with equal degrees are $v_{\left\lfloor\frac{n}{2}\right\rfloor}$ and $v_{\left\lfloor\frac{n}{2}\right\rfloor+1}$. Since, $1=d\left(v_{1}\right)<\cdots<d\left(v_{\left\lfloor\frac{n}{2}\right\rfloor}\right)=d\left(v_{\left\lfloor\frac{n}{2}\right\rfloor+1}\right)<\cdots<$ $d\left(v_{n}\right)=n-1$, we obtain

$$
\begin{aligned}
\operatorname{irr}_{t}\left(H_{n}\right) & =\sum_{i>j}\left(d\left(v_{i}\right)-d\left(v_{j}\right)\right)=\sum_{i=2}^{n-1} \sum_{j=1}^{i-1}\left(d\left(v_{i}\right)-d\left(v_{j}\right)\right) \\
& =\sum_{i=2}^{n-1} \sum_{j=1}^{i-1}(i-j)+\sum_{j=1}^{\left\lfloor\frac{n}{2}\right\rfloor-1}\left(\left\lfloor\frac{n}{2}\right\rfloor-j\right)+\sum_{i=\left\lfloor\frac{n}{2}\right\rfloor+1}^{n-1}\left(i-\left\lfloor\frac{n}{2}\right\rfloor\right) \\
& =\sum_{i=2}^{n-1} \sum_{j=1}^{i-1} j+\sum_{i=1}^{\left\lfloor\frac{n}{2}\right\rfloor-1} i+\sum_{i=1}^{\left\lceil\frac{n}{2}\right\rceil-1} i=\left(\begin{array}{c}
n \\
3
\end{array}\right)+\left(\begin{array}{c}
\left\lfloor\frac{n}{2}\right\rfloor \\
2
\end{array}\right)+\left(\begin{array}{c}
\left\lceil\frac{n}{2}\right\rceil \\
2
\end{array}\right),
\end{aligned}
$$


which immediately gives $\operatorname{irr}_{t}\left(H_{n}\right)=\frac{1}{12}\left(2 n^{3}-3 n^{2}-2 n+3\right)$ for odd $n$, and $\operatorname{irr}_{t}\left(H_{n}\right)=\frac{1}{12}\left(2 n^{3}-\right.$ $\left.3 n^{2}-2 n\right)$ for even $n$.

Proposition 2.3 If $T$ is a tree with $n$ vertices, then $\operatorname{irr}_{t}(T) \leq(n-1)(n-2)$. Moreover, equality holds if and only if $T$ is a star.

\section{Proof:}

Let $T$ be a tree that is not a star, with $u$ being a vertex with maximal degree. Consider a pendant vertex $v$ that is a adjacent to a vertex $w \neq u$. We remove the edge $v w$ from $T$ and add the edge $u v$. After this replacement, only the degrees of $u$ and $w$ are affected, namely, $d(u)$ increases by one while $d(w)$ decreases by one. Thus, the summands $|d(u)-d(w)|$ and $|d(w)-d(u)|$ in 2 both increase by two. Each of the $2(n-2)$ summands $|d(u)-d(x)|$ and $|d(x)-d(u)|$ with $x \neq u, w$ increases by one while each of $2(n-2)$ summands $|d(w)-d(x)|$ and $|d(x)-d(w)|$ with $x \neq u, w$ increases or decreases by one. Thus, we have $\operatorname{irr}_{t}(T-w v+u v) \geq \operatorname{irr}_{t}(T)+2+n-2-(n-2)=\operatorname{irr}_{t}(T)+2$. Therefore the largest total irregularity among the trees of order $n$ is only attained by the star, whose total irregularity equals $(n-1)(n-2)$.

\section{References}

[1] H. Abdo, N. Cohen, and D. Dimitrov. Graphs with maximal irregularity. Filomat, 2014, in press.

[2] Y. Alavi, A. Boals, G. Chartrand, P. Erdős, and O. R. Oellermann. $k$-path irregular graphs. Congr. Numer., 65:201-210, 1988.

[3] Y. Alavi, G. Chartrand, F. R. K. Chung, P. Erdős, R. L. Graham, and O. R. Oellermann. Highly irregular graphs. J. Graph Theory, 11:235-249, 1987.

[4] M. O. Albertson. The irregularity of a graph. Ars Comb., 46:219-225, 1997.

[5] F. K. Bell. On the maximal index of connected graphs. Linear Algebra Appl., 144:135-151, 1991.

[6] A. Bondy and U. S. R. Murty. Graph Theory. Springer Verlag, Berlin, 2008.

[7] Y. Caro and R. Yuster. Graphs with large variance. Ars Comb., 57:151-162, 2000.

[8] G. Chartrand, P. Erdôs, and O. R. Oellermann. How to define an irregular graph. Coll. Math. J., 19:36-42, 1988.

[9] G. Chartrand, K. S. Holbert, O. R. Oellermann, and H. C. Swart. F-degrees in graphs. Ars Comb., 24:133-148, 1987.

[10] G. Chartrand, M. S. Jacobson, J. Lehel, O. Oellerman, S. Ruiz, and F. Saba. Irregular networks. Congr. Numer., 64:197-210, 1988.

[11] L. Collatz and U. Sinogowitz. Spektren endlicher Graphen. Abh. Math. Sem. Univ. Hamburg, 21:6377, 1957. 
[12] D. Cvetković and P. Rowlinson. On connected graphs with maximal index. Publications de l'Institut Mathematique (Beograd), 44:29-34, 1988.

[13] D. Dimitrov and R. Škrekovski. Comparing the irregularity and the total irregularity of graphs. Ars Math. Contemp., 2014, in press.

[14] P. C. Fishburn. Packing graphs with odd and even trees. J. Graph Theory, 7:369-383, 1983.

[15] P. Hansen and H. Mélot. Variable neighborhood search for extremal graphs. 9. bounding the irregularity of a graph. DIMACS Ser. Discrete Math. Theoret. Comput. Sci., 69:253-264, 2005.

[16] M. A. Henning and D. Rautenbach. On the irregularity of bipartite graphs. Discrete Math., 307:1467-1472, 2007.

[17] D. E. Jackson and R. Entringer. Totally segregated graphs. Congress. Numer., 55:159-165, 1986.

[18] D. Rautenbach. Propagation of mean degrees. Electr. J. Comb., 11:N11, 2004. N11.

[19] D. Rautenbach and I. Schiermeyer. Extremal problems for imbalanced edges. Graphs Comb., 22:103-111, 2006.

[20] D. Rautenbach and L. Volkmann. How local irregularity gets global in a graph. J. Graph Theory, 41:18-23, 2002. 